\title{
Maurice Barrès et Joseph Bédier : une amitié idéologico-littéraire
}

Alain Corbellari

\section{OpenEdition}

1 Journals

Édition électronique

URL : http://journals.openedition.org/edl/1061

DOI : 10.4000/edl.1061

ISSN : 2296-5084

Éditeur

Université de Lausanne

\section{Édition imprimée}

Date de publication : 15 septembre 2017

Pagination : 87-110

ISBN : 978-2-940331-65-9

ISSN : 0014-2026

\section{Référence électronique}

Alain Corbellari, « Maurice Barrès et Joseph Bédier : une amitié idéologico-littéraire », Études de lettres [En ligne], 2 | 2017, mis en ligne le 15 septembre 2019, consulté le 17 décembre 2020. URL : http:// journals.openedition.org/edl/1061; DOI : https://doi.org/10.4000/edl.1061 


\section{MAURICE BARRÈS ET JOSEPH BÉDIER: UNE AMITIÉ IDÉOLOGICO-LITTÉRAIRE}

Dreyfusard, le médiéviste Joseph Bédier (1864-1938) n’avait apparemment guère de raison d'être ami avec Maurice Barrès. La Grande Guerre les a pourtant rapprochés en les faisant communier dans le culte de la grandeur française et les a même amenés à collaborer autour d'un livre que l'on attribue généralement au seul Barrès, mais dans l'élaboration duquel Bédier semble avoir joué un rôle important: Les Diverses Familles spirituelles de la France.

Le médiéviste Joseph Bédier a eu avec Maurice Barrès une relation où nationalisme et Moyen Âge se sont mêlés dans des proportions complexes. S'il n'est plus aujourd'hui connu du grand public que par son adaptation du Roman de Tristan et Iseut, véritable best-seller du roman d'amour français au $\mathrm{XX}^{\mathrm{e}}$ siècle, Bédier fut l'un des intellectuels phare de cette "III" République des lettres" remise en lumière par Antoine Compagnon, qui n’a d'ailleurs qu'effleuré dans son livre fameux la figure de Bédier ${ }^{1}$. Chez ce dernier, les écrivains du début du $\mathrm{XX}^{\mathrm{e}}$ siècle ont en effet pu apprécier à la fois l'écrivain, vulgarisateur incontournable de la littérature française du Moyen Âge, et le philologue, auteur de travaux qui rendaient à la France la gloire de ses origines littéraires. Ainsi, de même que l'œuvre de Barrès ne saurait se réduire à ses positions nationalistes, celle de Bédier ne tient pas tout entière dans le renouveau

I. Voir A. Compagnon, La Troisième République des lettres. Compagnon a par contre consacré tout un livre à l'un des maîtres de Bédier, Ferdinand Brunetière, pour des raisons d'ailleurs plus politiques que littéraires (A. Compagnon, Connaissez-vous Brunetière?). 
de points d'histoire littéraire. Le frémissement des plus belles pages de Barrès transcende ce que d'autres peuvent avoir d'idéologiquement pesant, de la même manière que le Roman de Tristan et Iseut de Bédier donne de la littérature médiévale une idée plus immédiatement poétique que ses ouvrages sur les fabliaux ou les chansons de geste. On a donc envie de dire que ces deux grandes figures de la vie intellectuelle française du tournant du $\mathrm{XX}^{\mathrm{e}}$ siècle étaient faites pour se rencontrer.

Il s'en faut pourtant de beaucoup que cette rencontre ait eu lieu spontanément. Républicain radical convaincu, Bédier avait été dreyfusard comme la plupart de ses collègues; Paul Meyer, l'homme qui avait démontré la falsification du bordereau, était même l'un de ses maîtres les plus directs, et il fréquenta assidûment, de 1903 à 1913, le salon de la marquise Arconati-Visconti, bienfaitrice de sa chaire au Collège de France, où il croisait hebdomadairement Jaurès, Joseph Reinach et Dreyfus lui-même ${ }^{2}$. La plus ancienne allusion à Barrès retrouvée dans sa correspondance figure d'ailleurs précisément dans une lettre à la marquise Arconati, datée du 22 juillet 1908: Bédier y rappelle avec satisfaction à sa mécène le jour où elle avait "conspué Barrès " ${ }^{3}$.

Du chemin s'est donc visiblement fait - et peut-être des deux côtés - lorsque l'on trouve dans la correspondance de Bédier, datée du 8 juin 1914, sa première lettre à Barrès, qui ne constitue donc que la deuxième allusion à l'écrivain de cet important corpus épistolaire (dont nous préparons l'édition, à paraître à la suite de notre édition de la correspondance entre Bédier et Gaston Paris aux Edizione del Galluzzo à Florence). Brève, cette première lettre de Bédier à Barrès mérite d'être intégralement citée:

Monsieur,

J'ai eu l'honneur, il y a quelques années, de vous être présenté par notre ami commun Jean Tharaud. C’est pourquoi j’ai pris la liberté d'aller hier chez vous, au boulevard Maillot: je désirerais en effet vous entretenir d'un certain projet, qui n'est pas indigne, peut-être, de vous

2. Sur le salon de la marquise Arconati, voir G. Baal, «Un salon dreyfusard».

3. «Et je me rappelle en cette occasion une si belle lettre de M. Monod, à vous adressée, que vous me faisiez lire un jour au Collège de France (le jour où vous avez conspué Barrès) : il vous disait tout ce que vous lui avez fait de bien" (Paris, Bibliothèque Victor Cousin, Ms. 263, f 58-60). 
être exposé: il me serait plus facile de vous en parler que de vous en écrire. Malheureusement on n'a pas su me dire à votre porte quand vous rentrerez à Paris. Puis-je vous demander de me faire savoir si votre retour est prochain et, en ce cas, s'il vous sera possible de me donner un rendez-vous?

Veuillez agréer, Monsieur, l'assurance de ma plus haute considération ${ }^{4}$. Rappelons que Jean Tharaud (1877-1952) et son frère Jérôme (18741953) avaient été les élèves de Bédier à l'École Normale Supérieure. Condisciples de Péguy (qui admirait fort le Roman de Tristan et Iseut de son maître Bédier ${ }^{5}$ ), ils avaient été des collaborateurs de la première heure aux Cahiers de la Quinzaine et se firent connaître en publiant ensemble des romans ${ }^{6}$, des chroniques et des recueils de contes souvent inspirés du Moyen Âge ${ }^{7}$. Or Jérôme a été secrétaire de Barrès de 1904 à la Grande Guerre, et il succédera à Bédier au trente-et-unième fauteuil de l'Académie française, où il sera reçu en 1940 par Georges Duhamel (Jean sera également accueilli chez les Immortels en 1946).

De quel projet Bédier voulait-il entretenir Barrès? Il est trop tôt, ce 8 juin, pour que l'idée ait un rapport avec la guerre, même imminente. Une lettre du 16 octobre 1913 à la marquise Arconati-Visconti nous apprend que Bédier avait espéré faire jouer durant la saison 1913-1914 la pièce qu'il avait tirée, avec son cousin Louis Artus, de son Roman de Tristan et Iseut (et qui ne sera finalement créée qu'en 1929). Le philologue aurait-il essayé d'attirer sur son projet l'attention de l'un des écrivains les plus célèbres de la France d'alors? Cela n'est pas impossible. Mais les relations entre les deux hommes ont finalement pris une autre tournure.

Rappelons par ailleurs qu'au moment où Bédier lui écrit, Barrès n'est pas encore rentré du voyage en Orient qu'il avait entrepris afin d'observer la place que tenait la France dans ces régions (et dont il ne tirera qu'en 1923 son Enquête aux pays du Levant). Parti le $1^{\mathrm{er}}$ mai, il ne sera de retour que le $1^{\text {er }}$ juillet, et se trouvait alors entre Beyrouth et Alep.

Une chose est sûre: l'heure n'est plus, désormais, pour Bédier au conspuage de Barrès. Si son Roman de Tristan et Iseut, paru en 1900, a

4. BnF, Fonds Barrès, Lettre de Bédier $\mathrm{n}^{\circ} 1$.

5. Voir A. Corbellari, Joseph Bédier écrivain et philologue, p. 138 sq.

6. Citons en particulier J. et J. Tharaud, Dingley, l'illustre écrivain, qui reçut le prix Goncourt en 1906.

7. J. et J. Tharaud, Les Contes de la Vierge. 
fait de lui un auteur connu du public cultivé, le travail qui a définitivement assis sa réputation de savant est ses Légendes épiques, qui reprennent les cours qu'il a donnés au Collège de France où il a été nommé en 1903. Avant-guerre, en effet, la France semble encore s'opposer à l'Angleterre dans ses prédilections médiévales conformément au partage décrit en 1200 par le trouvère arrageois Jean Bodel. Celui-ci disait - les médiévistes connaissent bien cette phrase - que la "matière de Bretagne", c'est-à-dire les contes arthuriens, était «vaine et plaisante» alors que la "matière de France", donc les chansons de geste, avaient la dignité de l'histoire même. Laissant la première aux Anglais (ou à la littérature, comme en témoigne son adaptation tristanienne), Bédier décrivait, dans Les Légendes épiques, les chansons de geste comme purement françaises, infléchissant et continuant à la fois la ligne de son maître Gaston Paris qui avait, en 1871, dans Paris assiégé, exalté La Chanson de Roland comme le récit prémonitoire d'une glorieuse défaite... finalement vengée. Terminant son grand ouvrage en 1913, Bédier avait ainsi immédiatement été salué comme le chantre d'un effort national dont l'objectif se faisait de plus en plus précis, et on imagine bien qu'il n'avait guère protesté contre cette lecture de son œuvre. La parenté entre les visions que Bédier et Barrès ont alors de la France est d'autant plus patente que les quatre volumes de Bédier sont construits autour de l'évocation des monastères où, selon lui, ont germé les chansons de gestes qui nous sont parvenues. Or ces lieux se nomment entre autres Vézelay, Saint-Gilles du Gard, Stavelot, Malmédy, Le Mont Saint-Michel... Bédier écrit ainsi à propos de légende de Renaut de Montauban:

Stavelot-Malmédy, abbaye de l'Ardenne et abbaye que se partageaient les diocèses de Liège et de Cologne, fut le lieu du monde le plus propre à fomenter à la fois des légendes ardennaises et des légendes rhénanes ${ }^{8}$.

Comment ne pas songer au fameux prologue de La Colline inspirée de Barrès où sont évoqués les «lieux où souffle l'esprit»? Ainsi y trouve-ton entre autres évoquée «la noire forêt des Ardennes, tout inquiétude et mystère, d'où le génie tira, du milieu des bêtes, ses fictions les plus aériennes" 9 .

8. J. Bédier, Les Légendes épiques, t. IV, p. 272 sq.

9. M. Barrès, La Colline inspirée, p. 273. 
Les deux textes sont de 1913! Il y a donc peu d'apparence que l'un ait inspiré l'autre, ce qui ne rend leur conjonction que plus frappante: il s'en dégage à l'évidence une même "idée de la France», comme le dira plus tard un autre grand Français. Barrès le savait d'ailleurs bien qui inscrivit cette dédicace (difficile à lire) dans l'exemplaire de La Colline inspirée qu'il offrit à Bédier:

2 juin 1914
A Joseph Bédier,
J'offre ce livre de mes promenades dans ce lieu digne des romans de chevalerie que sa science nous fait aimer et comprendre. Ah! si vous pouviez, mon cher ami, me dire quelles légendes on chantait à Sion- Vaudémont, ou mieux encore me mettre à même de retrouver quelque [?] de la sainte colline dans les romans de chevalerie, ou tout au moins me donner le moyen de m'adresser en [?], là-haut, dans les bois [???], Lorraine, [???], de Vaudémont [?] descendant des Carolingiens et ser- vant la [?] d'Huon Capet.
Maurice Barrès ${ }^{10}$

Ce qui nous ramène à la lettre écrite par Bédier, seulement six jours plus tard, à Barrès: on imagine mal que les deux hommes n'aient rien eu à se dire! Peut-être cependant la rencontre proposée n'eut-elle pas lieu, car la missive resta isolée. Et il faut attendre deux ans, plus exactement jusqu'au 3 juin 1916, pour voir la correspondance reprendre. Beaucoup de choses se sont passées entre les deux lettres: Barrès est devenu le «rossignol des carnages»; quant à Bédier, qui était apparemment l'un des rares universitaires français à savoir suffisamment l'allemand pour s'atteler à cette tâche, il avait publié en 1915 la brochure Les crimes allemands d'après des témoignages allemands, dont le retentissement immédiat avait amené la même année la publication d'un second opuscule, justificatif, Comment l'Allemagne essaye de justifier ses crimes ${ }^{11}$. Traduisant des carnets de soldats allemande morts ou prisonniers et tenant par là des documents sur les exactions de l'ennemi, Bédier souleva en effet une polémique

Io. Ouvrage conservé chez les héritiers Bédier.

II. Toutes deux parues chez Armand Colin dans la collection «Études et documents sur la Guerre", dans le comité de laquelle on retrouve Lavisse, Boutroux, Bergson, Andler, Durkheim, bref toute la «Troisième République des Lettres». 
européenne qui ne sera sans doute pas pour rien dans l'élaboration, après-guerre, de la notion de "crime de guerre».

Si la lettre de 1914 témoignait vraisemblablement d'un rapprochement entre deux écrivains, les suivantes, qui se succéderont à intervalles rapprochés à partir de 1916, réuniront clairement deux hommes qui combattent par la plume. La première missive de cet ensemble, celle du 3 juin, ne témoigne encore que de l'admiration de Bédier, mais on sent que celui-ci brûle de collaborer plus étroitement avec Barrès. Nous la citerons également in extenso:

Cher Monsieur,

Notre géographe militaire, que j’ai consulté dès hier soir, me dit: «Si les Italiens ne tiennent pas là où ils sont maintenant, il faudra qu'ils dégarnissent, et au plus vite, tout leur front Est, qu'ils abandonnent l'Isonzo, la Carnie, les Dolomites... Tiendront-ils? Il y a espoir que oui, rien de plus» - Je remarque que l'avisé Polybe, qui avait commencé une relation de son voyage, s'est arrêté après son premier et unique article. Pour l'instant, en l'état d'incertitude où nous sommes, la description admirative d'une région militaire si menacée risque, je le crains, de produire sur tout lecteur une impression pénible. Au contraire, comme vos notes de voyage reprendraient de l'accent, quelle ne serait pas leur valeur de sincérité, dans quelques jours ou semaines, s'il se confirme que le front par vous visité subsiste intact!... - Que votre article de ce matin sur la fête de Jeanne d'Arc, le finale surtout ${ }^{12}$, est bienfaisant et beau! Je vous enverrai d'ici à deux jours ce que je sais de Marguerite de Vaudémont: elle était l'arrière-petite-fille

I2. M. Barrès, "L'hommage national à Jeanne d'Arc». L'article se termine ainsi: "Sitôt que ses compagnons d'armes, ses frères et ses pareils, le glorieux et malheureux peuple des tranchées sera revenu de la guerre, Jeanne, par une promotion unanime, montera au faîte de notre vie nationale. Allons tous, demain, au pied de ses images, honorer en elle les absents, les meilleurs, les Français de la première classe, les soldats de la Grande Guerre» (L'intuition de Barrès était juste: Jeanne d'Arc sera canonisée en 1920). 
de mon cher Joinville ${ }^{13}$. Je vous remercie de votre si bonne lettre et vous prie de vouloir bien agréer mes sentiments d'entier dévoûment ${ }^{14}$.

La date de 1916 n'est pas précisée, mais elle se déduit des événements évoqués: Barrès était allé visiter le Front italien du 9 au 19 mai 1916; mettant ses notes au propre entre le 25 mai et le 27 juin de la même année, il devait en tirer en 1917 ses 10 jours en Italie. Quant au "géographe militaire», alias Polybe (pseudonyme dont il signait ses «Commentaires» journalistiques), c'est Joseph Reinach, que Bédier connaissait par le salon de la marquise Arconati. Visiblement, le fait que le Juif Reinach ait été l'un des dreyfusards les plus ardents ne pose plus problème à Barrès.

En octobre au plus tard, le vœu implicite de Bédier est devenu réalité: Barrès et lui travaillent de concert à un livre qui ne peut être que Les Diverses Familles spirituelles de la France ${ }^{15}$. La troisième lettre de Bédier, du 5 octobre, est particulièrement révélatrice:

Cher Monsieur,

J'ai été à Neuilly. Vous étiez parti de l'avant-veille. Mais on m’a mené à votre cabinet de travail, et j'ai déposé sur votre bureau le lot de livres et de brochures que je vous rapportais. J'y ai joint une douzaine de feuillets de mon écriture, où sont des extraits de ces volumes. Il y a là quelques bons textes: si vous vous occupez présentement de ce "tableau de nos vertus communes et variées", peut-être devriez-vous dire à un secrétaire de vous envoyer à Charmes les feuillets manuscrits et les deux ou trois volumes où il verra des signets de papier marquant certaines pages, - et aussi la brochure où sont les lettres du jeune

I3. Jean de Joinville (1225-1317), auteur de la fameuse Vie de saint Louis que Bédier évoquera en 1923 dans son chapitre sur les chroniqueurs médiévaux de la grande Histoire illustrée de la littérature française co-dirigée par lui et Paul Hazard, eut un fils, Anseau, qui épousa une Marguerite de Vaudémont et qui fut le grand-père d'une autre Marguerite, celle dont il est ici question: épouse de Ferry V de Lorraine (mort à Azincourt en 1415), elle sera en effet à l'origine de la seconde branche des comtes de Lorraine, dite de Lorraine-Vaudémont, qui tirent leur nom d'un lieu cher entre tous à Maurice Barrès puisqu'il est le théâtre de La Colline inspirée, où Marguerite n'est, de fait, pas citée.

I4. BnF, Fonds Barrès, Lettre de Bédier $n^{\circ} 2$.

15. M. Barrès, Les Diverses Familles spirituelles de la France. 
soldat protestant Cazalis ${ }^{16}$. Mon ami Andler ${ }^{17}$ m'a dit vous avoir vu; il avait l'air très content de son entretien avec vous; mais nous n'avons fait que nous croiser dans la rue, pressés l'un et l'autre, et nous n'avons pu causer. Je souhaite qu'il vous ait donné de beaux documents.

Puissent ces quelques jours de repos à la campagne vous être bienfaisants! Dès votre retour, puisque vous le voulez bien, j'irai vous voir, et je vous prie de vouloir bien accepter, en attendant, l'assurance de mon affectueux dévoûment ${ }^{18}$.

Cette lettre montre à l'évidence que la part de Bédier dans l'élaboration des Diverses familles spirituelles de la France est importante. Un passage de la sixième lettre, du $1^{\text {er }}$ décembre 1916, nous apparaît même encore plus révélateur:

Un «rabbin» et un «syndicaliste» ne pourraient-ils vous fournir des textes de citations à l'ordre du jour qui fassent pendant à ceux-là? S'il en existe quelques-uns, ils suppléeraient à la difficulté que vous avez à vous procurer de belles lettres de soldats israélites et de soldats socialistes.

Ainsi, Bédier semble-t-il bien être à l'origine de l'extension qu'a fini par prendre l'ouvrage de Barrès, puisque celui-ci a, on le sait, scrupuleusement suivi, les conseils de Bédier, qui à son tour peut bien être considéré comme le co-auteur du livre. Si Barrès a seul tenu la plume, le chantre des amours de Tristan en est selon toute probabilité l'inspirateur essentiel.

Nous reproduisons en appendice les autres lettres du philologue à l'écrivain. La dernière, en 1923, témoigne éloquemment - et cette constatation va nous permettre de passer de la politique à la littérature - de l'estime dans laquelle le grand styliste Barrès tenait l'universitaire Bédier. On le voit en effet, ayant consulté ce dernier sur l'usage d'un mot peu courant ("apiétrir»), recevoir de lui un véritable cours de grammaire et de stylistique. Et cela l'année de la mort de Barrès arrivé alors au faîte de sa gloire!

I6. Il n'est pas question de ce Cazalis dans le chapitre sur les protestants des Diverses familles spirituelles de la France.

17. Charles Andler (1866-1933), germaniste, biographe de Lucien Herr (La vie de Lucien Herr), auteur d'un fameux livre sur Nietzsche (Nietzsche, sa vie et sa pensée), fut professeur de langue et littérature germaniques au Collège de France à partir de 1925.

I8. BnF, Fonds Barrès, Lettre de Bédier $n^{\circ} 3$. 
Dès la fin de la Guerre, les relations des deux hommes s'étaient en effet poursuivies dans un cadre essentiellement littéraires. La parution en 1919 de L'Effort français de Bédier, réunion d'articles de propagande militaire, avait en effet tant enthousiasmé Barrès que l'auteur de $L a$ Colline inspirée s'était fait fort de faire élire celui des Légendes épiques à l'Académie française comme en témoigne une lettre de Barrès à Bédier datée du 9 mai 1919:

Mon cher ami,

Il faut que je vous dise d'un mot combien j'aime et j'admire «Notre infanterie" ${ }^{19}$. Je savais bien que les quelques pages de l'histoire de Port-Royal de Racine étaient d'un beau goût mais je ne comprenais pas la raison de cette sobriété. Sans doute que je n'avais pas en moi la piété qui avait commandé cette retenue à Racine. En vous lisant, je suis étroitement associé par avance à vos sentiments de vénération et je comprends de cour et d'esprit votre brûlante réserve d'expression. Votre ouvrage est d'une très grande beauté. On demande l'influence de la guerre sur les lettres: la guerre créera un public pour une telle pensée. Je ne vous ai jamais dit que je voudrais pour l'Académie votre entrée parmi nous, mais je l'ai dit à plusieurs de mes confrères et demain c'est tout le monde qui va nous reprocher de ne pas vous avoir déjà appelé. Vous n'avez qu'à continuer à ne pas vous préoccuper des textes neufs et sûrement ils vont vouloir vous faire le quarantième. J'ai un bien grand plaisir qu'un tel livre soit publié et par vous ${ }^{20}$.

Bédier avait été élu l'année suivante et avait prononcé son discours de réception le 3 novembre 1921.

Précisons que d'autres lettres de Barrès à Bédier dorment encore dans le fonds des héritiers Bédier, répondant essentiellement à celle envoyées par Bédier durant la Guerre. Comme elles n'éclairent pas davantage la relation que nous avons ici révélée, nous nous sommes permis d'en faire l'économie, mais on en trouvera une transcription complète dans l'édition que nous préparons.

Il nous faut maintenant aborder les rapports plus strictement littéraire qu'entretient l'œuvre de Barrès avec celle de Bédier. En 1922, Barrès publiait son dernier roman, Un jardin sur l'Oronte, et il n'est sans doute guère aventureux d'y lire un hommage au recréateur du Roman de

19. J. Bédier, "Notre infanterie».

20. Fonds des héritiers Bédier. 
Tristan et Iseut. Ainsi l'ouverture du roman se fait-elle sur un fond tristanien explicite, lorsque Guillaume dans les jardins de l'émir avoue qu'il vient d'entendre réciter la légende des amants de Cornouailles:

Dernièrement on nous a récité le merveilleux enchantement de Tristan et Iseult, et nous nous réjouissions à regarder de jeunes visages émus par les mêmes sentiments qui nous troublaient (Un jardin sur l'Oronte, p. 18).

Comme le fera Denis de Rougemont plus tard dans L'amour et l'Occident, Barrès semble se souvenir ici de la fameuse phrase de La Rochefoucault: "Combien de personnes seraient tombées amoureuses si elles n'avaient jamais entendu parler d'amour?»

Guillaume commence alors de narrer l'histoire de Tristan dont il compte tirer une leçon d'indulgence morale peut-être applicable à Barrès lui-même, qui se souvient probablement ici d'Anna de Noailles:

Il dit comment ces deux-là burent le philtre d'amour et s'aimèrent invinciblement à travers toutes les misères, et comment nous devons leur pardonner leurs fautes, parce qu'aucun de nous, jeune ou vieux, n'est sûr qu'il ne va pas rencontrer l'être dont il subira jusqu'à la mort la fascination (Un jardin sur l'Oronte, p. 19).

On sait par ailleurs que Barrès aura à se faire pardonner par ses admirateurs les plus nationalistes, d'avoir écrit avec le livre que l'on est en train de lire un roman si peu "engagé " politiquement!

Mais Guillaume n'en reste pas longtemps à l'histoire de Tristan et Iseut et se voit bientôt invité à raconter ses propres aventures. On ne s'étonnera pas que l'ouverture de son récit renchérisse, comme d'ailleurs déjà le passage que nous venons de citer, sur le célèbre prologue du roman de Bédier :

Ma mère m’a raconté des histoires de ceux qui se sont aimés jusqu'à la mort, d'un amour si irrésistible qu'ils l'avaient éprouvé avant même de s'être rencontrés, [...] (Barrès, Un jardin sur l'Oronte, p. 21).

Relisons Bédier :

Seigneurs, vous plaît-il d'entendre un beau conte d'amour et de mort? C'est de Tristan et d'Iseut la reine. Ecoutez comment à grand'joie, à 
grand deuil ils s'aimèrent, puis en moururent un même jour, lui par elle, elle par lui (Roman de Tristan et Iseut, p. 15).

À vrai dire, le texte de Barrès, avec son allusion à l'amour «de loin» nous renvoie également à la légende de la princesse lointaine, ce qui n'est pas davantage dû au hasard, puisque Bédier avait succédé à l'Académie française à... Edmond Rostand! (On se souvient que ce dernier avait mis en scène les amours du troubadours Jaufré Rudel et de la belle Mélissinde de Tripoli dans La Princesse lointaine, en 1895).

Mais ce sont malgré tout d'abord les accents tristaniens qui frappent dans le roman de Barrès, fussent-ils ironiques comme lorsque la Sarrasine dans son jardin enchanté donne à Guillaume le nom de l'amant d'Iseut:

- Sire Tristan, croyez-vous que nous sommes ici une suffisante collection de mandragores, de basilics et de turquoises, pour composer un philtre d'amour efficace?

Toutes se mirent à rire (Un jardin sur l'Oronte, p. 45).

C'est cependant très sérieusement que la plainte d'Oriante, à la fin du roman fait à la fois résonner le motif du jardin des amoureux (le locus amænus de la topique médiévale), celui de la folie et celui de la mort d'amour:

C'est toi que j'attendais au jardin de l'Oronte, avant ta venue, et que j'ai reconnu; toi que j'ai compris ne pouvoir pas écarter quand pour notre malheur, sois béni, tu réapparus, mon amour; toi qui viens follement de nous perdre et que jusqu'à la mort, si je dois te survivre, je conserverai dans mon cœur (Un jardin sur l'Oronte, p. 183).

Et le narrateur d'évoquer les "paroles caressantes et tragiques" par lesquelles les amants échangent «les secrets de l'amour et de la mort» (p. 184), nouvelle formule directement inspirée par Bédier.

Certaines images semblent même se rejoindre de manière plus précise. Lorsque Isabelle dit à Oriante:

Rappelez-vous ce que dit le poète: «Entraînée par le blanc coursier du jour et par la cavale noire de la nuit, la vie galope à deux chevaux vers le néant» (Un jardin sur l'Oronte, p. 138).

Cette vision pourrait bien être médiatisée par un passage du roman de Bédier où les amants séparées languissent l'un de l'autre : 
Mais, sans relâche, dans l'ardeur de la fièvre, le désir l'entraînait, comme un cheval emporté, vers les tours bien closes qui tenaient la reine enfermée; cheval et cavalier se brisaient contre les murs de pierre; mais cheval et cavalier se relevaient et reprenaient sans cesse la même chevauchée (Roman de Tristan et Iseut, p. 75).

Par ailleurs, le Guillaume de Barrès s'inspire également d'un autre héros exalté par Bédier. Lorsqu'il avoue:

Je me suis croisé pour faire de grandes choses, pour gagner mon paradis dans le ciel et sur la terre. J'espérais voir des anges avant même que de mourir (Un jardin sur l'Oronte, p. 138).

Le héros d'Un jardin sur l'Oronte fait à l'évidence référence à La Chanson de Roland, au héros de laquelle l'archange Gabriel lui-même tend la main au moment de sa mort. L'allusion est même plus précisément faite à Roland tel que Bédier le voit, car la thèse des Légendes épiques est que les chansons de geste s'expliquent moins par leur origine historique lointaine que par le climat de croisade (en Espagne puis en Terre Sainte) qui a présidé à l'écriture des textes qui nous sont parvenus aux $\mathrm{XI}^{\mathrm{e}}$ et $\mathrm{XII}^{\mathrm{e}}$ siècles. Insistant sur le fait que les routes du pèlerinage de Compostelle le long desquelles les récits épiques ont, selon lui, été élaborées étaient en même temps les chemins qu'empruntaient les soldats français pour aller reconquérir l'Espagne, Bédier fait des épopées françaises des œuvres de propagande, sur un fond de croisades que les guerriers authentiques de Charlemagne n'avaient guère connu. On ne résistera pas au plaisir de citer ce qui est sans doute le plus beau moment lyrique, en même temps que la page la plus célèbre des Légendes épiques de Bédier:

Avant que le poète de la Chanson de Roland eût écrit, déjà l'on parlait sur cette route de Charlemagne comme d'un héros et de Roland comme d'un martyr.

Comment le comprendre? Au commencement était la route. En tout pays, dans tous les temps, les hommes ont peuplé de légendes les routes vénérables. Ce chemin qui va devant eux vers la terre qu'ils désirent, qui donc, prévoyant leur désir, l'a jadis tracé pour eux, un dieu ou un héros, Hermès ou Héraclès? Et toujours les voyageurs savent la réponse ${ }^{21}$.

2I. J. Bédier, Les Légendes épiques, t. III, p. 366. 
Mais Roland n'est pas le seul héros épique qui a pu inspirer Barrès. En 1915, Bédier avait lui-même adapté pour le théâtre, avec un considérable succès de circonstance, un épisode du cycle de Guillaume d'Orange montrant le départ au combat et la mort de Vivien, le neveu du fameux «marquis au court nez» Guillaume, à qui Barrès n'a d'ailleurs sans doute pas pour rien emprunté le prénom du héros de son propre roman!

Les sources médiévales d'Un jardin sur l'Oronte s'avèrent donc multiples et il n'est pas toujours évident de les identifier de manière certaine. Ainsi lorsque Guillaume dit à l'émir:

Si l'on entend un rossignol on dit à son ami: «Écoute!» Si l'on a dessiné et planté un beau jardin, on est content que d'autres l'admirent par-dessus le mur (Un jardin sur l'Oronte, p. 22).

On peut bien sûr penser au chapitre XIII du roman de Bédier justement intitulé "la voix du rossignol» où celui-ci est précisément, imité par Tristan, le signe de reconnaissance par lequel Iseut identifie la venue de son amant. Mais on peut aussi songer au Lai du rossignol (ou du Laüstic) de Marie de France où le chant de l'oiseau sert de prétexte à la rencontre d'un amant et d'une dame mariée séparés par le mur mitoyen de leurs propriétés respectives. Enfin, et surtout, toute l'atmosphère orientale d'Un jardin sur l'Oronte semble trahir la lecture par Barrès d'ouvrages sur la littérature arabe et persane pour lesquels Bédier n'a pas dû lui être d'une grande aide.

Il est possible d'attribuer au Roman de Tristan et Iseut un rôle séminal dans la genèse du dernier roman de Barrès et ce de manière d'autant plus vraisemblable que, comme nous l'avons vu, l'amitié et l'admiration réciproque des deux écrivains fut profonde. En même temps, les souvenirs littéraux du texte de Bédier dans Un jardin sur l'Oronte sont pour aussi dire inexistants: Barrès est un suffisamment grand écrivain pour transformer ses sources en quelque chose qui lui est éminemment personnel et en particulier pour investir son roman de toute sa propre fascination pour les "Pays du Levant». On sait qu'il a par là influencé à son tour d'autres auteurs comme Montherlant (La Rose des sables) ou Pierre Benoît (La Châtelaine du Liban) et on peut même se demander si Bédier, écrivant en 1923 le chapitre dédié aux chroniqueurs du XIII siècle dans la grande Histoire de la littérature française illustrée qu'il a codirigée avec Paul Hazard chez Larousse, et qui est le seul chapitre qu'il $\mathrm{y}$ ait rédigé personnellement, ne constitue pas un hommage à Barrès qui 
disparaît cette même année. La façon, en particulier, que Bédier a de tenter de justifier Villehardouin dans la difficile affaire de la prise de Constantinople trahit une vision de la grandeur de l'effort des Croisés en Grèce qui a sans doute quelque chose de barrésien:

Les Français qui conquirent Constantinople semblent d'abord avoir mérité entre tous l'accusation d'impérialisme si souvent repris contre nous. Pourtant, il apparaît qu'il ne se sont jamais battus que persuadés, selon l'expression d'un compagnon de Villehardouin, Robert de Clari, que «la bataille estoit droiturière». Ainsi ont fait les Français de tous les temps ${ }^{22}$.

Une idée développée par Barrès en 1906 dans Le Voyage de Sparte entre d'ailleurs particulièrement en consonance avec celles de Bédier. Regrettant la démolition de la tour construite par les Croisés sur l'Acropole, lors de la quatrième Croisade, un voyageur polémique avec un pensionnaire de l'École française d'Athènes:

Le "miracle grec», c'est beau, mais le miracle français, je veux dire notre expansion au treizième siècle, ce n'est pas mal non plus ${ }^{23}$.

Or, Bédier dans les dernières années de sa carrière, travaillait à une synthèse sur le "Premier siècle des lettres françaises", et l'expression "miracle du XI $\mathrm{XI}^{\mathrm{e}}$ siècle" revient à plusieurs reprises dans les articles programmatiques publiés peu avant sa mort ${ }^{24}$.

Assurément, Bédier et Barrès se faisaient une "certaine idée de la France», d'un nationalisme dont ils surent in fine tous deux tempérer l'intransigeance politique par la mise au premier plan d'une culture et d'un art de vivre transmis par une littérature aimée entre toutes: adaptateur ou transpositeur, ils en figurèrent les fidèles gardiens.

Alain Corbellari

Universités de Neuchâtel et de Lausanne

22. J. Bédier, «En relisant Villehardouin», p. 313.

23. M. Barrès Le Voyage de Sparte, p. 193.

24. Voir J. Bédier, "Le Moyen Âge». 


\section{BIBLIOGRAPHIE}

\section{Textes}

BArrès, Maurice, Le Voyage de Sparte, Paris, Juven, 1906, repris in Euvres complètes, t. VII, Paris, Club de l'Honnête Homme, 1967.

_, La Colline inspirée, Paris, Plon, 1913, repris in Euvres complètes, t. VI, Paris, Club de l'Honnête Homme, 1966.

- 10 jours en Italie, Paris, Crès, 1917, repris in Chronique de la Grande Guerre, Paris, Plon, 1934, t. VIII, p. 120-202.

—, "L'hommage national à Jeanne d'Arc", repris in Chronique de la Grande Guerre, t. VIII, Paris, Plon, 1934, p. 208-13.

—, "Le discours de Champigny. Où en sommes-nous?", 3 décembre 1916, repris in Chronique de la Grande Guerre, Paris, Plon, 1936, t. IX, p. 66-73.

—, Les Diverses Familles spirituelles de la France, Paris, Émile-Paul, 1917, repris in Euvres complètes, Paris, Club de l'Honnête homme, t. VIII, 1966, p. 317-478.

—, Un jardin sur l'Oronte, La Chaux-de-Fonds, Nouvelle Bibliothèque, 1945.

BÉDIER, Joseph, Le Roman de Tristan et Iseut, Paris, Sevin \& Rey, puis Piazza, 1900 ; édition critique par Alain Corbellari, Genève, Droz, "Textes littéraires français», 2012.

—, Les Légendes épiques, 4 vol., Paris, Champion, 1906-1913.

—, Les crimes allemands d'après des témoignages allemands, Paris, Armand Colin, «Etudes et documents sur la Guerre», 1915.

-, Comment l'Allemagne essaye de justifier ses crimes, Paris, Armand Colin, «Etudes et documents sur la Guerre», 1915.

—, "Notre infanterie», Revue des Deux Mondes, 50 (1919), p. 771-802 et 51 (1919), p. 52-75, repris dans L'Effort français, Paris, CalmannLévy, 1919, p. 1-145.

—, "Les chroniqueurs», in Histoire illustrée de la littérature française, éd. en collaboration avec Paul Hazard, Paris, Larousse, t. I, p. 75-84. 
—, "En relisant Villehardouin", Revue de France, 2 (1923), p. 711-721, repris in Philologie et humanisme, articles et préfaces inédits en volume, éd. par Alain Corbellari, Paris, Classiques Garnier, 2010 ("Recherches médiévales», 4, Série «Magistralia. Leçons et lectures", 1).

—, "Le Moyen Âge», in L'Encyclopédie française, t. XVI «Arts et littératures dans la société contemporaine", Paris, comité de l’Encyclopédie française éditeur, 1935, p. 16'10-3 - 16'10-9, repris in Philologie et humanisme, op. cit., p. 25-36.

—, "La poésie française aux jours de la Première Croisade», Revue des Deux Mondes, $\mathrm{CVI}^{\mathrm{e}}$ année, huitième période, tome XXXVI, 15 décembre 1936, p. 809-22, repris in Philologie et humanisme, op. cit., p. 39-51.

—, Correspondance avec Gaston Paris, éd. par Ursula Bähler et Alain Corbellari, Firenze, Edizioni del Galluzzo per la Fondazione Ezio Franceschini, «L'Europe des philologues», 1, 2009.

Bescherelle aîné, Dictionnaire national ou Dictionnaire universel de la langue française, 2 vol., Paris, Garnier, 1845-1846.

Tharaud, Jérôme et Jean, Dingley, l'illustre écrivain, Paris, Pelletan, 1906.

—, Les Contes de la Vierge, Paris, Plon, 1940

\section{Travaux}

Andler, Charles, La vie de Lucien Herr, Paris, Rieder, 1932.

—, Nietzsche, sa vie et sa pensée, 6 vol., Paris, Bossard, 1920-1931.

BAAL, Gérard, "Un salon dreyfusard, des lendemains de l'Affaire à la Grande Guerre, la marquise Arconati-Visconti et ses amis", Revue d'histoire moderne et contemporaine, 28 (1981), p. 433-463.

Compagnon, Antoine, La Troisième République des lettres. De Flaubert à Proust, Paris, Seuil, 1983.

-, Connaissez-vous Brunetière? Enquête sur un antidreyfusard et ses amis, Paris, Seuil, "L’Univers historique», 1997.

Corbellari, Alain, Joseph Bédier écrivain et philologue, Genève, Droz, "Publications romanes et françaises», 1997.

Rey, Alain, Littré. Le lexicographe et les mots, Paris, Gallimard, 1970. 


\section{APPENDICE \\ AUTRES LETTRES DE BÉDIER À BARRÈS \\ (BNF, FONDS BARRÈS)}

Lettre $n^{\circ} 4$

mardi

[24. 10. $1916^{25}$ ]

Cher Monsieur,

Je n'ai reçu qu'hier soir votre lettre. Malheureusement je serai pris cet après-midi par les formalités de l'enrôlement de mon fils cadet. Je tâcherai de faire demain mercredi la course de Neuilly, si du moins je ne suis forcé de le mener dès demain à son régiment, à Orléans. De toutes façon, ma semaine sera très chargée de besognes. Que votre entrée en matière est large et belle! que je vous remercie de me l'avoir communiquée! J'ai grand hâte de connaître la suite. Veuillez accepter, cher Monsieur, mes sentiments d'affectueux dévoûment.

J. Bédier

\section{Lettre $n^{\circ} 5$}

Paris mardi [fin 1916 26]

Cher Monsieur,

Ce matin, j'avais une conférence à Sèvres ${ }^{27}$. C'est cet après-midi que j'espérais pouvoir aller à Neuilly: une affaire est venue à la traverse, qui m'appelle à la mairie à 5 h. $1 / 2^{28}$. Recevant votre lettre à l'instant, j’aurais écarté cette affaire: mais voici que je viens sottement de me luxer le bras droit (de là l'écriture que vous voyez), ce qui me fait un peu souffrir. Mais demain mercredi je serai vraiment libre, et (sauf avis contraire de

25. Le timbre de ce pneumatique est difficilement lisible, mais l'année est assurée par l'allusion à l'enrôlement de Jean Bédier, né en 1898; et le 24 octobre était bien un mardi.

26. Seul le classement de cette lettre dans le Fonds Barrès permet d'en inférer la date approximative.

27. À l'École Normale Supérieure de jeunes filles.

28. Rappelons que Bédier était adjoint à la mairie du $\mathrm{V}^{\mathrm{e}}$ arrondissement. 
votre part) j'arriverai chez vous de bonne heure dans la matinée (vers 9 h., 9 h. $1 / 2$ ?). Je ne soupçonnais aucunement que vous attachiez du prix à ces conversations, et que mon retard pût retarder votre travail. Si je l'avais mieux compris ou senti, je me serais débrouillé pour venir plusieurs fois la semaine dernière ${ }^{29}$. Mais désormais je tâcherai de réparer... je n'ai pas de désir plus ardent que de vous servir en quelque chose, si peu que ce soit, sentez-le bien - et veuillez croire à mon très reconnaissant dévoûment.

J. Bédier

\section{Lettre $n^{\circ} 6$}

Paris, 11 rue Soufflot

$1^{\text {er }}$ déc. 1916

Cher monsieur,

Je viendrai certainement, sauf avis contraire de votre part, vous voir lundi matin; mais comme je vous ai vu un peu inquiet l'autre jour au sujet de votre prochain article ${ }^{30}$, je viens vous en dire tout de suite mon sentiment; et, comme je vois que vous ne craignez pas que la poste égare vos manuscrits, je vous retourne sous ce pli les feuillets que vous m'aviez remis ou envoyés.

Ces pages sont de toute beauté: sauf deux ou trois détails notés sur les papiers ci-joints, je n'y vois rien à changer dans les documents, ni dans les commentaires.

Quant aux citations des prêtres à l'ordre de l'armée ${ }^{31}$, je pense tout à fait comme vous: il convient d'achever l'énumération de ces textes. Les sacrifier, ce serait donner à tous l'impression qu'ils sont en très petit

29. Ce détail nous fait entrevoir un aspect tout à fait méconnu de la vie de Barrès: apparemment ses relations avec Bédier furent pendant une période assez longue au moins hebdomadaires. La correspondance est trop pauvre, malheureusement, pour que l'on puisse reconstituer même le cadre des conversations entre les deux hommes, mais il est certain que Bédier était devenu pour Barrès un confident non négligeable et même - l'honneur n'est pas mince de la part d'un auteur si vanté pour son style! - une référence en matière stylistique, comme le montrera très bien la lettre $\mathrm{n}^{\circ} 10$, du 13 mars 1923.

30. M. Barrès, «Le discours de Champigny. Où en sommes-nous?» est le seul article recensé entre le 23 novembre et le 17 décembre 1916: commençant par un hommage à Paul Déroulède, Barrès y fait le bilan guerrier de l'année 1916.

3I. Bédier revient ici à l'élaboration des Diverses familles spirituelles de la France. 
nombre. Le petit scrupule littéraire qui vous gêne est, je crois, bien peu de choses en présence de textes si essentiels. Le plus simple, pour vous débarrasser de ce scrupule, serait peut-être de l'avouer très simplement à vos lecteurs au début de l'article: c'est l'esprit et l'intention des quelques lignes que vous trouverez ci-jointes (feuillets $a$ ); si vous récriviez ce petit "chapeau» en disant plus explicitement encore l'embarras que vous avez ressenti, ce serait parfait.

Un «rabbin» et un «syndicaliste» ne pourraient-ils vous fournir des textes de citations à l'ordre du jour qui fassent pendant à ceux-là? S'il en existe quelques-uns, ils suppléeraient à la difficulté que vous avez à vous procurer de belles lettres de soldats israélites et de soldats socialistes.

Veuillez agréer, cher Monsieur, l'assurance de mon affectueux dévoûment.

Jh Bédier

\section{Lettre $n^{\circ} 7$}

[cachet postal: 20.12.16]

Cher monsieur,

Sauf avis contraire de votre part, je viendrai vous voir demain jeudi dans la matinée (étant malheureusement pris vendredi et samedi). Encore faudra-t-il que je sois rentré à la rue Soufflot vers midi, parce que dès 1 heure je dois être au théâtre Sarah-Bernhardt (représentation organisée par la municipalité de mon arrondissement ${ }^{32}$ ). C'est pourquoi je me permettrai de sonner à votre porte d'assez bonne heure (vers $9 \mathrm{~h}$. $1 / 4)$ ? Si cette combinaison vous gêne trop, je pourrai aussi bien venir demain vers 4 heures - en quittant le théâtre.

Veuillez accepter, cher Monsieur, l'assurance de mon dévoûment bien affectueux.

Jh Bédier

32. Bédier parlerait-il d'une reprise de sa pièce Chevalerie, adaptée de la légende de Guillaume d'Orange? Dans ce cas, il faudrait ajouter cette représentation à la liste que j'en donne en p. 385 de mon Joseph Bédier écrivain et philologue. Mais il peut aussi bien s'agir d'une représentation de n'importe quelle pièce à laquelle Bédier était tenu d'assister, de par ses fonctions officielles d'adjoint au maire du $\mathrm{V}^{\mathrm{e}}$ arrondissement. 


\section{Lettre $n^{\circ} 8$}

[cachet postal: 22. 1. 1917]

Cher monsieur,

je suis, ces jours-ci tellement encombré par mes besognes que je ne sais comment y échapper; pour quelques semaines encore il me faudra vivre sous ce tunnel. J'ai pourtant des choses à vous dire sur les lettres de l'Alsacien Meyer: je viendrai donc boulevard Maillot aussitôt que je pourrai, avant la fin de cette semaine. Quant à vos articles sur les "Familles spirituelles", je les ai lus un à un de très près sans y trouver une phrase, un mot que je voulusse vous proposer de changer: ils sont, dans le détail comme dans l'ensemble, admirables et bienfaisants. Mais je vous demande de me faire la faveur de m'en envoyer une épreuve en placards, dès qu'ils seront réimprimés: pour la première fois alors, je verrai l'ensemble, les proportions, le mouvement général, et c'est alors seulement que je pourrai peut-être vous soumettre quelque remarque utile. Pour le moment, j'ai été au plus pressé, qui était de vous envoyer (ce que j'ai fait hier) quelques citations propres à étoffer votre article sur Albert Thierry (mais que ce livre d'Albert Thierry est médiocre et décevant!) ${ }^{33}$

Veuillez agréer, cher Monsieur, l'assurance de mon bien affectueux dévoûment.

J. Bédier

\section{Lettre $n^{\circ} 9$}

18 février 1919

Cher monsieur et grand ami,

Ce mot vous sera remis par Lorentz Eckhoff, de Christiania, qui est le traducteur de votre Colette Baudoche ${ }^{34}$ : il a donc déjà un titre à

33. Albert Thierry (1881-1915), pédagogue de tendance anarchiste, mort au Front. L'allusion de Bédier s'éclaire à la lecture des Diverses familles spirituelles de la France de Barrès, p. 376: "J'ai lu dans les Entretiens des non-combattants (mai-juin, 1916, 21, rue Visconti) les carnets où Albert Thierry, instituteur syndicaliste et le plus violemment sincère des syndicalistes, crayonnait comme un testament ses suprêmes pensées de politique et de morale et recherchait quelle justice doit être réalisée dans le monde pour que la paix définitive s'établisse».

34. Lorentz Eckhoff (1884-1974), historien de la littérature norvégien, avait également traduit, en 1918, Le Roman de Tristan et Iseut de Bédier. Il a écrit (en 
votre aimable accueil; mais il en a un plus grand encore, celui d'aimer beaucoup notre pays et de le servir utilement de son influence. Je suis sûr que vous aurez de la joie à causer avec lui. Veuillez agréer, cher Monsieur et ami, mon plus affectueux souvenir.

Joseph Bédier

Lettre $\mathrm{n}^{\circ} 10^{35}$

Paris, 11, rue Soufflot

13 mars 1923

Cher grand ami,

Je ne suis pas grand clerc en matière de grammaire. Voici pourtant quelques réflexions.

Apiétrir ne semble pas attesté jusqu'à maintenant. Votre lexicographe, Bescherelle ${ }^{36}$ ou un autre, a pu le rencontrer dans quelque texte moderne, sous la plume d'un homme de lettres qui essayait de le lancer. Il y a eu des dizaines de milliers d'essais de ce genre: celui-ci, il est constant qu'il a avorté.

Pourtant, le mot est correctement formé. Si beaucoup d'adjectifs (chauve, par exemple) n'ont pas de verbe correspondant, un plus grand nombre a servi à former des verbes en -ir: pauvre, appauvrir, - vil, avilir, - couard, accouardir; etc.

Pourrait-on aujourd'hui sur ce type «relancer» le mot avec quelque chance de succès?

$\left.1^{\circ}\right)$ Ce serait bien tard, à la onzième heure. La conjugaison en -ir ne semble plus très vivante. On a formé récemment rosir et amerrir (lequel du reste offre un cas particulier: il a été favorisé par l'analogie d'atterrir). Mais ce sont les deux seuls verbes récents en -ir qui me reviennent à

norvégien) un livre sur Verlaine et le symbolisme (1923), ainsi que des études sur Shakespeare (1939) et sur Gide (1947). On trouve jointe à la présente lettre une adresse probablement écrite de la main même d'Eckhoff: «Lorentz Eckhoff / 51 rue Claude Bernard, Paris / Slottetsgate 31, Christiania".

35. Cette lettre est accompagnée, dans le Fonds Barrès de la BnF, d'une copie dactylographiée très fautive destinée au t. 34 des Cahiers de Maurice Barrès.

36. Bescherelle aîné, Dictionnaire national ou Dictionnaire universel de la langue française. Ce dictionnaire estimable, que Bédier semble considérer comme une vieillerie, avait en effet été détrôné par le Littré, d'ailleurs cité plus bas par Bédier. 
l'esprit. On dit "accouardir", parce qu'on le dit depuis des siècles; on ne songerait pas à former un verbe «alâchir».

$2^{\circ}$ ) «S'apiéttir», réfléchi, supposerait un apiétrir, actif, dont le sens se voit mal: «rendre quelqu'un piètre»?

$\left.3^{\circ}\right)$ De blême, on a tiré blêmir, qui est compris d'emblée. On conçoit donc qu'apiétrir, tiré de piètre puisse l'être aussi. Cependant la transformation nécessaire de l'e ouvert de piètre en l'e fermé de apiétrir cause une certaine gêne, requiert de celui qui entend apiétrir un certain effort pour qu'il comprenne.

$\left.4^{\circ}\right)$ Le sens, à son tour, s'impose-t-il d'emblée? Piètre (= pedestris, très probablement) est un adjectif curieux, qui par lui-même ne suggère pas un sens bien défini, une image bien nette (au rebours de chétif ou mesquin, qui sont les mots par lesquels Littré le traduit). Le mot piètre a si peu de substance qu'il est presque toujours employé comme épithète: il tire presque toute sa valeur du substantif qui l'accompagne (un piètre mari, un piètre personnage). Ou si on l'emploie comme attribut, il faut ajouter une détermination qui fait ressortir ce qu'il y a d'accidentel dans l'état désigné par le mot: «il a été piètre ce jour là, en telle circonstance», mais on ne dira pas «il est piètre».

On dira: "C'est un piètre mari", mais on ne dira guère "Ce mari est piètre» (à moins d'y mettre l'intonation très juste et seulement à condition d'éclaircir la phrase par ce qui précède).

De même pour les choses. On dira, "Cette compensation est piètre" (il s'agit d'un cas particulier), mais on ne dira guère: Cette table est piètre (comme on dirait qu'elle est laide).

En somme, à mon sens - mais je suis Grosjean qui en remontre à son curé $^{37}$, - un écrivain d'aujourd'hui peut l'employer, mais à condi-

37. Comprendre: «je suis un ignare qui prétend apprendre quelque chose à son maître». La clause de modestie est presque obligée s'adressant à un auteur considéré comme le plus grand styliste de son temps. De fait, si Bédier offre ici une belle leçon de sensibilité à la langue et à cette notion évanescente qu'est l'«usage» (leçon tout à fait digne d'un authentique écrivain), il se montre en revanche — osons le dire — piètre lexicographe! Sa confiance en Littré est parfaitement aveugle et s'il avait ouvert le Bescherelle, il y aurait trouvé le mot. Surtout, il aurait pu ouvrir le Grand Larousse $d u X I X^{\mathrm{e}}$ siècle qui en donne la définition suivante: "rendre piètre, chétif, affaiblir: le manque d'exercice apiétrit promptement les enfants. // ce mot a vieilli.» [sic!] Le Quillet, en 1965, l'écrit avec deux p, ne faisant par là que suivre Furetière qui le glosait, en 1690, comme un terme de marchands (nous voyons ici, en passant, la confirmation d'une remarque d'Alain Rey qui s'étonnait, dans Littré. Le lexicographe et les mots, de 
tion qu'il le prépare, qu'il l'encadre de synonymes, qu'il s'efforce d'y faire entrer bon gré mal gré une image. Ce néologisme pourra être le bienvenu au lecteur de telle page de cet écrivain; mais il n'y a guère de chances que ce lecteur le recueille, s'en serve à son tour, et que le mot entre dans l'usage courant.

Respectfully submitted ${ }^{38}$. Je vous remercie, mon cher Maître et ami, de m’avoir donné cette petite occasion de vous prier d'accepter l'assurance de mon fidèle et profond attachement.

Joseph Bédier

ce que Littré n'avait apparemment jamais ouvert le Dictionnaire de Furetière). Le Trésor de la langue française, enfin, donne une référence de 1675, confirmant la définition de Furetière: apiétrir était un mot utilisé dans la langue commerciale pour parler des marchandises dont la valeur s'est amenuisée. Loin, donc, d'être un néologisme, comme le croit Bédier, le mot semble être tombé en désuétude dès le XVIII siècle. Quant à s'apiétrir, le Trésor de la Langue Française en donne un exemple tiré d'un recueil poétique publié en 1844 par A. Pommier, Les Colères. En fait, le seul point sur lequel Bédier ne se trompe pas est que le mot ne s'est jamais réellement imposé dans la langue (le Trésor de la Langue Française concède en effet qu'il est "peu usité»).

38. "Respectueusement soumis». Cet anglicisme désinvolte surprend sous la plume de Bédier (et d'autant plus en pareil contexte!), mais il en dit sans doute long sur la familiarité de Barrès et de l'auteur des Légendes épiques. 
